\title{
Análisis De La Praxis Pedagógica Y Su Contribución Al Modelo De Desarrollo Socioeconómico De Venezuela
}

\section{Analysis Of The Pedagogical Praxis And Its Contribution To The Model Of Socioeconomic Development Of Venezuela}

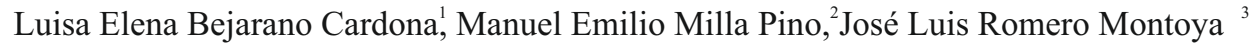

\begin{abstract}
RESUMEN
El trabajo tuvo como objetivo Analizar la Praxis Pedagógica que realizan los docentes en la Universidad Politécnica Territorial de Yaracuy "Arístides Bastidas" (UPTYAB), Venezuela, en términos de lo establecido para el modelo de producción contemplado en el Primer Plan Socialista Desarrollo Económico y Social de la Nación (PPSDESN) 2007-2013 y el Segundo Plan Socialista de Desarrollo Económico y Social de la Nación (SPSDESN) 2013-2019, el tipo de investigación es analítica apoyada en un diseño no experimental, observacional, descriptivo de campo, la muestra la conformaron 27 docentes y 236 estudiantes pertenecientes a los cuatro primeros PNF que se implementaron en la UPTYAB, el análisis de la información se realizó usando la estadística no paramétrica a través del análisis univariado y el de ACPC para un $\mathrm{P}<0,05$ obteniendo como resultado que la praxis pedagógica que realizan los docentes sometidos a este estudio, se desarrolla entre los estilos de enseñanza tradicional-constructivista-humanista, por lo que se concluye que además de estos estilos de enseñanza, se ha de requerir en la praxis docente, de la didáctica crítica como práctica de la investigación, para alcanzar en mayor medida los objetivos establecidos para el modelo de producción social contemplado en los PDESN 2007- 2013 y 2013- 2017.
\end{abstract}

Palabras clave: Praxis pedagógica, modelo de desarrollo, enseñanza tradicional.

\section{ABSTRACT}

The aim of the study was to analyze the Pedagogical Praxis performed bu teaches at the Polutechnic Territorial University of Yaracuy "Aristides Bastidas" (UPTYAB), Venezuela, in terms of what was established for the production model contemplated in the First Socialist Plan Economic and Social Development of the Nation (PPSDESN) 2007-2013 and the Second Socialist Plan for Economic and Social Development of the Nation (SPSDESN) 2013-2019, the type of research is analytical supported by a non-experimental, observational, descriptive design of the field, the sample was composed 27 teachers and 236 students belonging to the first four PNF that were implemented in the UPTYAB, the analysis of the information was made using the nonparametric statistics through the univariate analysis and the ACPC for a $\mathrm{P}<0.05$, obtaining as a result that the pedagogical practice that the teachers undergo in this study, develops between traditional teaching styles-co nstructivist-humanist, so it is concluded that in addition to these teaching styles, it must be required in the teaching praxis, critical didactics as a practice of research, to achieve in greater measure the objectives established for the social production model contemplated in the PDESN 2007-2013 and 2013-2017.

Keywords: Pedagogical praxis, development model, traditional teaching

\footnotetext{
${ }^{1}$ Universidad Politécnica Territorial de Yaracuy “Arístides Bastidas” - Venezuela, Autor de correspondencia:bejaranol@hotmail.com

${ }^{2}$ Universidad Nacional "Toribio Rodríguez de Mendoza" de Amazonas, Perú
} 


\section{INTRODUCCIÓN}

Las metas primordiales que el estado Venezolano tiene planteadas, en el camino hacia la consolidación del socialismo del siglo XXI, es básicamente la superación del modo de producción capitalista y del neoliberalismo como aparato ideológico político que lo sustenta por un modo de producción más humano.

En este sentido, el gobierno nacional ha hecho esfuerzos para tratar de transformar el modo de producción capitalista a otro donde se pueda superar el desequilibrio ecológico, la pobreza, la exclusión social y garantizar a todos los ciudadanos el pleno disfrute de sus derechos sociales básicos, es decir que considere lo humano, que el fruto del trabajo vaya en beneficio de la sociedad, donde el desarrollo económico esté en equilibrio con el ambiente y que las riquezas sean repartidas con justicia.

De allí que el Estado Venezolano pone en marcha el Proyecto Nacional "Simón Bolívar Primer Plan Socialista de Desarrollo Económico y Social de la Nación (PPSDESN) (2007-2013).

Este plan propone una transición del modelo capitalista rentista petrolero, al modelo socialista, cuyas característica son: a) impulsar un desarrollo endógeno como base económica del Socialismo del siglo XXI b) incrementar la soberanía y seguridad alimentaria c) fomentar la ciencia y la tecnología al servicio del desarrollo nacional d) reducir la diferencia en el acceso al conocimiento e) desarrollar la industria básica no energética, la manufactura y los servicios básicos. (República Bolivariana de Venezuela, 2007).

Reflexionando sobre los párrafos anteriores, podríamos preguntarnos ¿cómo hacer para que este marco legal se desarrolle y comience a dar los resultados propuestos? Si existen las bases legales y políticas ¿qué se necesita para comenzar a disfrutar de los buenos propósitos de este modelo productivo socialista?

Una de las posibles respuestas podría ser a través de la educación; pues no hay duda de que le corresponde a la educación el papel protagónico de formar a los hombres y mujeres que guiaran los destino de esta patria, para que de esta manera, lo contemplado en el marco legal del PPSDESN (2007-2013), correspondiente al modelo de producción, no se quede en letra muerta.

Es así que para darle continuidad a lo establecido en este primer plan socialista PPSDESN (2007-2013), se diseña el Segundo Plan Nacional de Desarrollo Económico y Social mejor conocido como Plan de la Patria (SPSDESN) 2013-2019 (República
Bolivariana de Venezuela, 2013), donde en su objetivo específico 2.2.2.9. Señala adecuar el sistema educativo al modelo productivo y en el 2.2.2.11 que establece la transformación universitaria en torno a la formación integral, la producción intelectual y la vinculación social que garantice el desarrollo de las potencialidades creativas y capacidad resolutiva frente a los problemas prioritarios del país.

Como se observa, ambos objetivos vinculan el proceso de enseñanza a la formación de un hombre y una mujer con conocimientos aptitudes y actitudes cónsonas con un modelo de producción que garantice la conservación del ambiente, desarrollando programas y proyectos que den respuesta a las necesidades productivas de gran importancia para el desarrollo de las comunidades y por ende de nuestro país.

Para lograr estos objetivos, la educación ha de formar hombres y mujeres integrales en lo científico, lo técnico, en lo humano, en lo moral, en lo estético en lo político, aptos para ser útiles a sí mismos y a la sociedad, para ser factores de cambio y desarrollo social.

De allí que Lanz citado por Quevedo (2010), señale que la educación, la ciencia y la tecnología deben recuperar su pertinencia social, estableciendo una relación armónica con la naturaleza, fortaleciendo el desarrollo endógeno y promoviendo una cultura de la solidaridad.

En atención a lo antes dicho, Prieto Figueroa (1970), expresaba que la educación dibuja el régimen que la soporta, afirmando que si el Estado es fascista la educación es fascista, si el Estado es democrático la educación debe ser democrática, si el Estado es socialista la educación debe ser socialista.

Basándonos en lo expresado por el Maestro Prieto (ob. cit) se infiere que la educación es una actividad social que tiene como expresión final la formación de hombres y mujeres, capacitándolos para que actúen como motor del cambio social; de allí, que educación y sociedad estén intrínseca y dialécticamente interrelacionadas en un proceso en el que la educación se organiza fundamentándose en las características del cuerpo social y éste a su vez, se ve influido por el producto que genera el acto educativo.

Se reafirma entonces la necesidad de que la educación venezolana esté vinculada con los planes nacionales de desarrollo económico y social de la nación, (PPSNDESN) (2007-2013) y el (SPSDESN) $(2013$ - 2019) para que de esta manera se pueda desarrollar el modelo de producción tal y como lo señala Álvarez (2009), un modelo de producción que abra amplios caminos para el empoderamiento 
popular y la masiva inclusión social en función del desarrollo humano integral, para la adopción y difusión de los valores de solidaridad, cooperación, complementación, reciprocidad, equidad y sustentabilidad.

En este orden de ideas, la CRBV (1999), señala en sus artículos 102 al 104, que toda persona tiene derecho a una educación integral de calidad; es decir, la educación es una actividad social que tiene como expresión final la formación del hombre para el mundo de la vida en sociedad, capacitándolo para que actúe como motor fundamental en las transformaciones sociales.

En este mismo sentido, la Ley Orgánica de Educación (LOE), en su artículo 15 señala que la educación tendrá como fin desarrollar el potencial creativo de cada ser humano para el pleno ejercicio de su personalidad y ciudadanía, en una sociedad democrática basada en la valoración ética y social del trabajo liberador y en la participación activa, consciente, protagónica, responsable y solidaria, comprometida con los procesos de transformación social (Gaceta Oficial de la República Bolivariana de Venezuela, 2009)

El rango legal de los principios citados, deja claramente establecido que el estado y la sociedad venezolana, esperan que la educación forme un sujeto que desde el contexto del perfil ciudadano que se plantea, pueda mantener, desarrollar y perfeccionar la vida en una sociedad democrática; los artículos en cuestión establecen el tipo de hombre que hay que formar y el tipo de sociedad para la cual hay que formarlo.

Por su parte, en Venezuela la Educación Universitaria, como último segmento educativo, viene realizando cambios que permitan armonizar la estructura administrativa y académica con lo establecido en el PPSDESN y en el SPSDESN. En esta etapa de cambios, que están ocurriendo en la educación venezolana, se inscribe la Misión Alma Mater, orientada a profundizar y ampliar la transformación en la Educación Universitaria desde su estructura organizativa, académica y política, lo cual exige un esfuerzo extraordinario de las comunidades académicas, encaminado a cambiar sus modos de pensar y de actuar para desaprender y aprender nuevas concepciones tendientes a imprimir cambios sustanciales en las prácticas y procesos educativos.

Todo esto requiere colocar en el centro del debate lo referente a los docentes que han orientado históricamente la creación de conocimientos, su forma de enseñar y aprender para contribuir con la formación integral de profesionales que puedan ser capaces de actuar conscientemente en la creación, transformación y desarrollo del modelo de producción propuesto en los planes de la nación de nuestra sociedad venezolana, un individuo acorde a los requerimientos nacionales, es decir, la formación integral de profesionales con profundo sentido de país y al servicio de los intereses de la nación.

De allí que si partimos de la afirmación de que el docente es un mediador necesario en el proceso de enseñanza y que, cuanto mejor formado esté, desarrollará su trabajo eficientemente, resultará entonces un reto su praxis pedagógica para hacer posible la formación de los profesionales integrales que impulsen el desarrollo del modelo de producción antes descrito y establecido en los planes de desarrollo económico y social de la nación.

Ahora bien en relación a la praxis pedagógica, para Batista citado por, (Ruíz J. , 2012) "la praxis pedagógica es el quehacer coordinado de acciones y recursos que contribuye a potenciar el proceso didáctico que realizan los profesores para direccionar su práctica al cumplimiento de los propósitos educativos".

En este sentido, la educación universitaria cobra mayor importancia, pues es allí donde se forman los futuros profesionales, por tal motivo, la praxis pedagógica que desarrollan los docentes en el aula de clases redundara de manera significativa en los egresados de nuestras universidades y en particular los de la Universidad Politécnica Territorial de Yaracuy "Arístides Bastidas (UPTYAB), para lograr las transformaciones, el desarrollo económico y social de nuestro país.

La UPTYAB, (Gaceta Oficial de la República Bolivariana de Venezuela, 2014), a través de sus bases Epistemológicas, Filosóficas y Axiológicas establecidas en cada uno de los Programas Nacionales de Formación (PNF), está diseñada para contribuir con la consolidación del modelo de producción social que ayude a romper con el individualismo, los desequilibrios ambientales y a resolver los problemas prioritarios que padecen las comunidades, es decir una enseñanza acorde con las exigencia y necesidades de la sociedad para la cual los está formando.

Por lo expuesto en los párrafos anteriores y tomando como base la definición de Batista (ob.cit), para la presente investigación se plantearon las siguientes interrogantes ¿cómo es la praxis pedagógica en el claustro universitario? ¿Hay diferencias entre la praxis pedagógica de los docentes que laboran en los programas de formación que se imparten en la 
UPTYAB? ¿En qué medida la praxis pedagógica se ajusta a lo establecido en los planes de desarrollo económico y social de la nación en lo referente al modelo de producción para la sociedad venezolana?

\section{MATERIAL Y MÉTODO}

Desde el punto de vista epistemológico, la investigación sobre la praxis pedagógica que realizan los docentes en términos de lo establecido en el modelo de producción de los PSNDESN (20072013), se enmarcó en el paradigma cuantitativo. En cuanto al diseño es no experimental, observacional, descriptivo y de campo.

\section{Diseño muestral}

Docentes: En este estudio, la muestra es la misma población ósea 27 docentes que laboran con unidades curriculares anualizadas en los trayectos II y IV de los PNF en Agroalimentación (PNFA), Administración (PNFAD), Informática (PNFI) y Procesos Químicos (PNFPQ) en la UPTYAB, sede IUTY; por lo cual, según Hernández, Roberto, Fernández y Pilar, (2007) la muestra es censal, distribuidos según la siguiente tabla 5:

Estudiantes: Con relación a los estudiantes, el diseño muestral según Scheaffer y Mendenhall, (1986), corresponde a la porción de la población que se toma para realizar el estudio; de allí que la toma de la muestra se realizó a través de la técnica de muestreo estratificado. Dado que la población de estudiantes es conocida, se aplicó la fórmula para el tamaño de la muestra cuyo valor fue de $n=236$

\section{Técnicas de recolección de datos}

Para obtener la información de los docentes, se diseñó un instrumento, cuyo nivel de medición es Ordinal pues se estudian variables categóricas, similar a la escala de Lickert con cinco (5) alternativas de respuestas, donde cada una de ellas, tiene un puntaje asignado, que está en concordancia con la intensidad de la actitud, de allí que (5) se refiere a una opinión más positiva sobre la concepción que poseen de las bases filosóficas que soportan la malla curricular de los PNF, y la praxis pedagógica que desarrollan para la enseñanza de los contenidos correspondientes a las unidades curriculares, que administran en el respectivo PNF al que están adscritos; mientras que el puntaje más bajo (1) refleja una opinión más negativa sobre los aspectos antes mencionados, (Hernández,
Fernández, et al, 2007)

En este mismo sentido, se elaboró un segundo instrumento aplicado a una muestra representativa de estudiantes que son atendidos por los mismos docentes sometidos al estudio.

El instrumento que se aplicó a los estudiantes, también fue un cuestionario tipo Lickert con cinco alternativas de respuesta todo esto con el propósito, de indagar sobre la praxis pedagógica que los docentes desarrollan para la formación de los futuros profesionales que egresan de la UPTYAB.

La validez de los instrumentos que se aplicaron a los docentes y a sus estudiantes, se determinó a través del juicio de expertos Hernández, Roberto, Fernández, et al (ob,cit).

La confiabilidad de ambos instrumentos, se determinó a través del cálculo del Coeficiente Alpha de Cronbach; el valor obtenido haciendo uso del SPSS v20.0 para $\alpha$ fue de: 0,898 descrito como una magnitud muy alta en la escala. De esta forma se constató que el instrumento diseñado era válido y confiable para ser aplicado a la población de estudio. En el caso de los estudiantes, para la aplicación de la prueba piloto, obtuvieron como resultado a través del SPSS v.20.0 un $\alpha$ de: 0,777 lo cual indica una alta confiabilidad del instrumento (Mongomery y Runger, 2003) y (Ruíz, ob.cit)

\section{RESULTADOS Y DISCUSIÓN}

\section{Análisis de los datos}

El análisis de la información obtenida a través de los instrumentos aplicados a los docentes y sus estudiantes, en un primer momento se realizó por medio del análisis univariado; en este sentido, por tratarse de instrumentos medidos con una escala tipo Likert se trabaja con el coeficiente de Correlación de rangos de Spearman para variables cualitativas ordenadas; con la finalidad de explorar el grado de asociación de las variables.

Para verificar la significancia estadística en lo referente a los ítem formulados a las muestras de docentes y estudiantes sometidos a estudio; en cuanto a las categorías de respuestas obtenidas, se aplicó la técnica de Correlación de Rango de Spearman debido a que los datos están medidos en una escala ordinal, seleccionando aquellas correlaciones cuyo valor sea significativos, es decir un valor de $\mathrm{P}<0,05$ lo que demuestra desde el punto de vista probabilístico la 
relación existente entre los ítems formulados, respecto a las categorías de respuestas obtenidas.

Una vez seleccionadas aquellas correlación de Spearman con $\mathrm{P}<0,05$ se utiliza una segunda prueba que permiióa medir de manera conjunta la independencia entre los ítem seleccionados de las muestras de docentes y estudiantes sometidos a estudio en cuanto a las categorías de respuestas obtenidas. Se aplicó la prueba de independencia de Ji-Cuadrado.

Con el propósito de analizar cuáles de los ítems impactan con mayor relación a las categorías de respuestas obtenidas, se usa la metodología de Análisis Multivariante, el cual permite profundizar la información obtenida al reducir aquellas variables que realmente no están relacionada de manera directa, para así obtener las que si están más directamente relacionada con el fenómeno que se está investigando, esto se logra al utilizar la técnica denominada Análisis de Componente Principal Categórico (ACPC).

\section{Análisis de los datos obtenidos del instrumento aplicado a los docentes}

De acuerdo al grado de asociación encontrada estadísticamente para un $\mathrm{P}<0,05$ se interpreta que de los 35 items que posee el instrumento, sólo $10 \mathrm{de}$ ellos son los que más ayudan a revelar la concepción que sobre el currículo poseen los docentes encuestados en la UPTYAB y de la praxis pedagógica que desarrollan en esa misma universidad. Se pudo evidenciar en conjunto que las diez variables presentan una significativa asociación, sin embargo, para conocer la relación de dependencia o de independencia entre estas variables se realizó la prueba de contingencia a través de un Jicuadrado.

El análisis $\mathrm{Ji}$ - cuadrado revela que los docentes encuestados tienen conocimiento de que la implementación del plan de estudio de los PNF contribuye con la formación socialista basada en la práctica de la solidaridad, la lucha contra el individualismo, la corrupción y el burocratismo, pero en su praxis pedagógica, la clase magistral es la estrategia usada para el desarrollo de los contenidos de la unidad curricular que administras.

\section{Análisis de los datos obtenidos de la}

\section{muestra de estudiantes}

Para el estudio de la información obtenida del instrumento aplicado a la muestra de los estudiantes, se aplicó al igual que para los docentes la Prueba de Correlaciones de Rango de Spearman, para explorar el grado de asociación de las variables (ítems); donde de 22 de ellas se obtuvieron sólo seis.

A la luz de los resultados anteriores, la evidencia estadística desde el análisis univariado (Respuestas Categóricas-Ítems), indicó que los items que aportan mayor información son las variables (items) 8,10 y 11. Lo que permite inferir que los docentes parten de los conocimientos previos que los alumnos poseen antes de desarrollar un nuevo conocimiento, aceptan sus sugerencias y/o aportes y toman en cuenta sus problemas y/o necesidades en el desarrollo de la praxis pedagógica que realizan.

El Análisis de Componentes Principales Categórico (ACPC) es la técnicas de análisis multivariado que se usó para identificar las dimensiones más relevantes sobre la praxis pedagógica de los docentes en la UPTYAB, pues es una técnica cuyo punto fuerte es la síntesis de la información o reducción de la dimensionalidad (número de variables) a partir de la creación de dimensiones del conjunto de variables observada.

Para la interpretación de los resultados se obtuvo la matriz de saturaciones la cual permitió relacionar las variables con los componentes, obteniéndose un conjunto de variables relacionadas entre sí, las cuales se interpretaron para generar en conjunto el nuevo componente.

En este orden de ideas, se analizó la praxis pedagógica que desarrollan los docentes encuestados en esta investigación con base en un conjunto de dimensiones o factores que midieron en docentes y estudiantes, los diversos aspectos de los que se compone ese fenómeno.

Para el análisis de fiabilidad interna en el ACPC, se utilizó el coeficiente de Alfa de Cronbach, bajo el Software SPSS 20 (2011) cuyo valor fue: 0,965 lo que se considera alto, es decir es el grado de explicación que permite los ítems en relación a las respuestas de interés; también se observó como las dimisiones 1 y 2 aportan un Alfa de Cronbach 0,931 y 0,843 lo cual se considera alto, serían entonces, los grados de explicación de los ítems en relación a las respuestas de interés por cada una de las dimensiones. 
Tabla Resumen de Saturación Items y Dimensiones (Docentes)

\begin{tabular}{|c|c|c|}
\hline Ítems & Dimisión 1 & \\
\hline $\mathrm{X} 1$ & $\begin{array}{l}\text { Organizas jornadas, charlas, foros entre otros, que promuevan la vinculación de la } \\
\text { universidad con las comunidades. }\end{array}$ & 0,814 \\
\hline $\mathrm{X} 4$ & $\begin{array}{l}\text { Cuando desarrollas los contenidos en la unidad curricular que administras haces } \\
\text { hincapié en la formación ciudadana más que en la formación meramente técnica y de } \\
\text { pensamientos instrumentalizados. }\end{array}$ & 0,862 \\
\hline $\mathrm{X} 6$ & $\begin{array}{l}\text { Planificas las clases preparando actividades o recursos en correspondencia con el } \\
\text { proyecto sociocomunitario, sociointegrador o sociotecnológico. }\end{array}$ & 0,879 \\
\hline X13 & $\begin{array}{l}\text { Para la enseñanza de los temas de la unidad curricular promueves el uso de las } \\
\text { Tecnologías de comunicación e información (TIC). }\end{array}$ & 0,897 \\
\hline $\mathrm{X} 15$ & $\begin{array}{l}\text { Vinculas los contenidos de la unidad curricular con otras disciplinas del conocimiento, } \\
\text { a través de ejemplos concretos de la realidad cotidiana de las comunidades, del estado } \\
\text { o del País. }\end{array}$ & 0,885 \\
\hline $\mathrm{X} 21$ & $\begin{array}{l}\text { Refuerzas los temas vistos en la unidad curricular realizando visitas a la comunidad, } \\
\text { empresas o instituciones relacionadas con el proyecto sociointegrador, } \\
\text { sociocomunitario, y/o sociotecnológico según sea el caso. }\end{array}$ & 0,783 \\
\hline $\mathrm{X} 25$ & $\begin{array}{l}\text { Con los conocimientos adquiridos, los egresados de los PNF se integraran a las } \\
\text { comunidades para lograr el desarrollo endógeno. }\end{array}$ & 0,774 \\
\hline $\mathrm{X} 35$ & $\begin{array}{l}\text { La implementación del plan de estudio de los PNF contribuye con la formación } \\
\text { socialista basada en la práctica de la solidaridad, la lucha contra el individualismo, la } \\
\text { corrupción y el burocratismo. }\end{array}$ & 0,726 \\
\hline Ítems & Dimisión 2 & \\
\hline X32 & $\begin{array}{l}\text { Con el desarrollo de la malla curricular de los PNF se logra afianzar en los futuros } \\
\text { egresados valores que resguarden la identidad nacional y la construcción de una } \\
\text { sociedad igualitaria. }\end{array}$ & 0,799 \\
\hline
\end{tabular}

Fuente: Bejarano (2016)

Interpretación: Los ítem que cumplen con el criterio establecido, generan dos grupos homogéneos que interactúan entre sí, tanto intro/entre dimensiones ( 1 y2), por lo que el aporte que revelan son las estrategias de enseñanza y el uso de las TIC para la formación de un egresado más humano.

A continuación se presentas los análisis realizados a la información obtenida de los estudiantes:

El análisis de fiabilidad interna para el Análisis de Componentes Principal Categórico (ACPC), se

Utilizó el coeficiente de Alfa de Cronbach, bajo el Software SPSS 20 (2011). Se observó en el total de Alfa de Cronbach que existe un 0,940 por lo que se considera alto, es decir es el grado de explicación que permite los ítems en relación a las respuestas de interés, de esta manera vemos como las dimisiones 1 y 2 aportan un Alfa de Cronbach 0,907 y 0,586 lo cual se considera alto para la dimensión 1 y buena para la dimensión 2 , serían los grados de explicación de los ítems en relación a las respuestas de interés por cada dimensión.

\begin{tabular}{|c|c|c|}
\hline Ítems & Dimensión 1 & \\
\hline $\mathrm{X} 8$ & $\begin{array}{l}\text { Los Docentes toman en cuenta los conocimientos que los estudiantes poseen antes de } \\
\text { desarrollar el nuevo contenido del tema o programa de la unidad curricular. }\end{array}$ & 0,706 \\
\hline $\mathrm{X} 10$ & $\begin{array}{l}\text { En las clases, los Docentes aceptan sugerencias o aportes que realizan los estudiantespara } \\
\text { el desarrollo de los contenidos. }\end{array}$ & 0,755 \\
\hline $\mathrm{X} 11$ & $\begin{array}{l}\text { Para la Enseñanza de los contenidos de la unidad curricular, los docentes toman encuenta } \\
\text { los problemas y/o necesidades de los estudiantes }\end{array}$ & 0,736 \\
\hline X19 & $\begin{array}{l}\text { En las clases los docentes los incentivan para que cuando egresen del PNF conformen } \\
\text { empresas de producción social. }\end{array}$ & 0,739 \\
\hline $\mathrm{X} 20$ & $\begin{array}{l}\text { En el desarrollo de las clases los docentes los incentivan a fomentar la ciencia y la } \\
\text { tecnología para el desarrollo del País. }\end{array}$ & 0,740 \\
\hline $\mathrm{X} 22$ & $\begin{array}{l}\text { En las clases los docentes les incentivan a valorar las relaciones con el ambiente, con la } \\
\text { naturaleza y con el trabajo solidario y liberador.. }\end{array}$ & 0,759 \\
\hline \multirow[t]{2}{*}{ Ítems } & Dimensión 2 & \\
\hline & ------ & \\
\hline
\end{tabular}

Fuente: Bejarano (2016) 
Interpretación: los ítem que cumplen con el criterio establecido, generan solo un grupo homogéneo que interactúan entre sí, en este sentido, el grupo de la dimisión 1 es el que aporta mayor información la cual como se evidencia son las estrategias de enseñanza.

Para el logro del tercer objetivo específico relacionado con la indagación del estilo de docencia que realizan los profesores en cada uno de los Programas Nacionales de Formación (PNF) investigados, se consideró la dependencia entre dichos Programas y los Items, que desde el punto estadístico del análisis univariado, son los que aportan mayor información, ellos son: 7, 8, 10, 11, $12,16,21,26$, utilizando la prueba Ji-cuadrado.

\section{CONCLUSIONES}

En lo referente al primer objetivo, la evidencia estadística desde el análisis univariado (RespuestasCategóricas-Ítems), reveló que los docentes encuestados tienen conocimiento en que la implementación del plan de estudio de los PNF contribuye con la formación socialista basada en la práctica de la solidaridad, la lucha contra el individualismo, la corrupción y el burocratismo, pero en su praxis pedagógica, la clase magistral es la estrategia usada para el desarrollo de los contenidos de la unidad curricular que administras.

En este sentido se concluye que éstos docente poseen una clara concepción de las bases filosóficas del currículo establecido en los Programas Nacionales de Formación (PNF) puesto que allí se contempla que la relación profesor-estudiante, ayuda en el establecimiento de una relación dialógica, sustentada en la cooperación, la solidaridad, la equidad, el interés mutuo y la corresponsabilidad, principios filosóficos que se deben desarrollar mediante el contacto realizado con sus estudiantes para la formación integral de los futuros egresados. Esto de igual forma fue manifestado por los estudiantes cuando señalaron que los docentes aceptan sus sugerencias y/o aportes y toman en cuenta sus problemas y/o necesidades en el desarrollo de la praxis pedagógica que realizan.

En cuanto al segundo objetivo específico se determinó que los docentes en su praxis pedagógica, utilizan principalmente la clase magistral como estrategia de enseñanza, lo que nos permite inferir que no implementan la didáctica crítica lo que dificultaría la participación activa del alumno limitando su criticidad aspecto de mucha importancia para la transformación social y su tributación con los planes de desarrollo económico y social de la nación (PDESN) 2007-2013 y 2013-2019

Lo anterior se sustenta ya que Schaller (citado por Olaya, ob.cit), indica que la didáctica crítica establece la enseñanza y el aprendizaje como una dualidad inseparable para introducir al discente en un proceso de formación que les permita solucionar sus problemas haciendo buen uso de su libertad, priviligiando la creatividad, solidaridad, cooperación y el cultivo de los valores humanos.

En lo refiere al tercer objetivo específico, desde el punto de vista de las pruebas estadísticas aplicadas, se reveló que la praxis pedagógica que utilizan los profesores del PNF en Agroalimentación tiene una tendencia a un estilo de enseñanza TradicionalContructivista-Humanista y un porcentaje alto en la dimensión relacionada con la formación del futuro egresado, especificamente en la conformación de empresas de producción social, aspecto de mucha importancia ya que es una de las directrices establecidas en el PDESN (2007-2013) para el modelo de producción social.

Para el caso del PNF en Administración, los docentes presentan un estilo de enseñanza TradicionalHumanista, lo que permite concluir que la praxis desarrollada por los docentes sometidos a este estudio poco tributa al modelo de producción propuestos en los PDESN. En este mismo estilo de docencia se ubican los docentes del PNF en Informática.

Finalmente para el PNF en Procesos Químicos estadisticamente se evidencio que predomina el estilo de enseñanza Constructivista -Humanista estilo que podría contribuir con la formación de un egresado acorde con las directrices comtempladas para el desarrollo del modelo de producción social planteado en los PDESN 2007-2013 2013-2019.

Por lo tanto, en atención a las respuestas obtenidas de los objetivos específicos de este trabajo, se concluye que la práxis pedagógica que han realizado los docentes en la UPTYAB, poco tributa con los lineamientos contemplados para el modelo de producción social establecidos en los PDESN (20072013 y 2013-2019), en virtud de que estos docentes a pesar de que conocen las bases filosóficas de la malla curricular de los PNF hacen poco uso de herramientas tales como la didáctica crítica, con técnicas como la enseñanza basada en problemas, todo esto con el firme propósito de lograr la formación de estudiantes capaces de impulsar los cambios de nuestra sociedad dando cumplimiento con el modelo de producción social.

Es así que para lograr los cambios y transformaciones que la sociedad venezolana reclama; debemos disponer de una educación que forme a los individuos 
acorde a las exigencias; esto es un ciudadano, que posea un nivel de criticidad para tomar las decisiones que favorezcan a la colectividad, por encima de las individualidades, un profesional integral e innovador.

En este sentido, se infiere que la praxis pedagógica que desarrollan los docentes, ha de requerir de mayores niveles de innovación y de preparación de tal manera que forme a los profesionales que la sociedad necesita; por ello, Bigott, (2011) señala que para dar respuestas a los problemas que demanda la sociedad venezolana, los jóvenes que se están formando en las instituciones de Educación Universitaria, deben recibir una educación integral que responda a las necesidades sociales y primordialmente vinculado con el modelo de producción propuesto en el Proyecto Nacional Simón Bolívar Primer Plan Socialista Desarrollo Económico y Social de la Nación (PPSDESN) 20072013 y el de la Ley Plan de la Patria Segundo Plan Socialista de Desarrollo Económico y Social de la Nación (SPSDESN) 2013-2019.

De igual forma a través del ACPC se reveló que las estrategias de enseñanza deben ajustarse a las establecidas en la malla curricular de los PNF, de tal manera que puedan vincularse con los PDESN 2007 2013-2019 y con ello la formación de un egresado más humano; éste resultado, fue corroborado por los estudiantes al develarse a través de ésta misma técnica que el estilo de enseñanza constructivista es el usado por los docentes en la praxis que desarrollan para la formación de los futuros profesionales.

\section{RECOMENDACIONES}

De acuerdo a los hallazgos obtenidos en este estudio, se considera que para darle vida a lo establecido sobre el modelo de producción en los PDESN para Venezuela, se recomienda que lo docentes reflexionen sobre la praxis pedagógica que están desarrollando, lo cual pueden lograr haciendo uso de herramientas tales como la sistematización de su trabajo académico e implementando diversos estilos de enseñanza.

Se debe formar al hombre para el mundo de la vida; con capacidad de criterio porque así lo reclama la actual relación universidad-sociedad. En la universidad de hoy, los sujetos deben aprender a construir, vigilar, controlar y ejercitar su propio y permanente autodesarrollo; entendiendo este proceso personal como un proceso dialéctico de acción de vida, que lo interrelaciona con el proceso de evolución sociohistórica de la humanidad.

Es muy importante que los que realizan la acción pedagógica se conviertan en docentes investigadores; es decir, vincular la teoría, la práctica y la investigación como una unidad indisoluble que permita abordar el claustro universitario a través del análisis que lo lleva a entender el contexto, la institución y el aula con el fin de construir estrategias didácticas coherentes y propiciadoras del desarrollo integral de dicho profesor, de sus colegas y de sus estudiantes.

El docente o la docente investigador(a), debe entender que no puede seguir actuando en el aula promoviendo el dogmatismo, viendo o desarrollando los contenidos de las unidades curriculares, como desconectados unos con otros, y como si fuesen aspectos ahistóricos y descontextualizados; por el contrario, la didáctica crítica plantea la conversión de este tipo de docente por un educador interesado en cuestionar permanentemente el proceso de enseñanza y de aprendizaje logrando así una conciencia crítica de su praxis pedagógica, al someter su ejercicio a continua reflexión y vigilancia con el fin de desarrollar la investigación en su praxis educativa.

Para Carr y Kemmis (1988), una didáctica crítica se adquiere haciendo uso de la investigación acción en el seno de la enseñanza, lo cual se puede realizar si se utilizan ciertas características que hagan crítica a la educación tales como: a) visión dialéctica de la realidad, b) usar la crítica ideológica para superar las interpretaciones distorsionadas, c) creando comunidades autorreflexivas que garanticen la unión de la teoría con la práctica.

Como se puede evidenciar el uso o la implementación de la didáctica crítica amerita de un docente bien formado tanto en el ámbito profesional como en el pedagógico para que de esta manera pueda hacer uso de la investigación acción en el corazón de su praxis pedagógica, desarrollando así una actitud crítica en sus estudiantes dando respuestas a las distintas problemáticas que se presentan en el entorno social que rodea a sus discentes, todo en el marco de lo establecido para el modelo de producción contemplado en los planes de la nación de nuestro país.

\section{BIBLIOGRAFÍA}

AnalyticalAlvares, V. (2009). Venezuela ¿Hacia donde va el Modelo Productivo? Caracas: Centro Internacional Miranda. Software (2003). User's Manual Statistix ${ }^{\circledR} \quad 8.0$. Tallahassee, Florida, U.S.A.

Bermejo, V. (2012). Análisis de los Cambios en la Metodología del Docente Universitario. Tesis de Maestria. Segovia, España: Magiisterio de Segovia. 
Bigott, L. (2011). Plan Nacional de Formación Permanente de Docentes Universitarios. Caracas : Ediciones de la Universidad Bolivariana de Venezuels.

Bruner, J. (2001). Enseñar a Aprender. Mexico: Mc Grawhill.

Carr, W y Kemmis,S (1988). Teoría Crítica de la Enseñanza: la investigación acción en la formación del profesorado. Barcelona España. Ediciones Martínez Roca.

Constitución de la República Bolivariana de Venezuela CRBV(1999), C. d. (15 de Febrero de 2009). CRBV(1999). Caracas, Venezuela: Mcultural.

Freire, P. (27 de 10 de 2007). Idoneos.com. Recuperado el 7 de 6 de 2008, de Idoneos.com:http://freire.idoneos.com/ind ex.php/319078.

Gaceta Oficial de la República Bolivariana de Venezuela. (3 de noveiembre de 2014). Decreto 1224 Creación de la Universidad Politécnica Territorial de Yaracuy "Arístides Bastidas". Caracas, Venezuela.

Gaceta Oficial de la República Bolivariana de Venezuela, (14 de mayo de 2008). $N^{\circ}$ 38.930. Programas Nacionales de Formación. Caracas, Venezuela: Ediciones de la Presidencia de la República.

Gaceta Oficial de la República Bolivariana de Venezuela. (Sabado 15 de Agosto de 2009). Ley Orgánica de Educación. Ley Orgánica de Educación. Caracas, Venezuela.

García, L. (2010). La Práxis del Docente Universitario y sus Retos ante la Construcción de la Condición Humana. Congreso Iberoamericano de Educación Metas 2021, (pág. 17). Buenos Aires.

Garza J, Morales B, González B. (2013). Análisis Estadístico Multivariante: Un Enfoque Teórico y Práctico. Primera Edición. Editorial Mc Graw-Hill Interamericana. Pp (331-364).

Gonzalez, O. (1999). Didáctica Universitaria. La Habana. Cuba: Universidad de la Habana

Hernández y otros. (2003). Metodología de la Investigación. México: Mc Graw Hill.

Hinkelarmmenrt. (1996). Una sociedad en la que todos quepan. San José: Duque José Editor.

Hurtado, J. (1998). Metodología de la Investigación Holística. Caracas: Fundación SYPAL.

Lanz, C. (sábado de febrero de 2006). Aporrea. Obtenido de Hacia la construcción de un nuevo estado y una nueva humanidad: http://www.aporrea.org/ideologia/a94671.h tml
Lanz, C (2006). Aportes para el Debate del Socialismo del Siglo XXI. Tesis.

Manual del Usuario. (2011). Sistema Básico IBM $®$ SPSS $®$ Statistics 20

Ministerio del Poder Popular para la Educación Universitaria. (24 de marzo de 2009). Misión Alma Mater. Decreto 6.650 Gaceta oficial $N^{\circ}$ 39.148. Caracas, Venezuela.

Montgomery, D y Runger, G. (2003). Probabilidady Estadística: Aplicada a la Ingienería. Mexico: 1era Edición Editorial Mc Grawhill.

Olaya, Alí. (2011) 4 Foro del Futuro Didáctica Crítica. Fondo Editorial Ipasme Caracas Venezuela .

Orcajo, A. (1998). La postmodernidad o la Fractura de las Emociones. Valencia Venezuela: Universidad de Carabobo.

Pérez. C (2004). Técnica de Análisis Multivariante de Datos. Aplicaciones con SPSS.

Prieto Figueroa, L. B. (1970). El Estado Docente en Venezuela. Caracas: Alianza.

Prieto Figueroa, L. B. (1970). El Estado Docente en Venezuela. Caracas: Alianza.

República Bolivariana de Venezuela. (2007). Proyecto Nacional Simón Bolívar Primer Plan Socialista PPS 2007-2013. Caracas: Presidencia de la República.

República Bolivariana de Venezuela. (4 de Diciembre de 2013). Ley Plan de la Patria II Plan Naconal de Desarrollo Económico y Social 2013-2019. Gaceta oficial N ${ }^{\circ} 40.308$ Extraordinario $N^{\circ} 6118$. Caracas, Venezuela: Ediciones de la Presidencia de la República.

Runger, G. y. (20003). Probabilidad y Estadística: Aplicaciones a la Ingieneriía. Mexico: Mc Grawhill.

Salinas, J. (2004). Innovación docente y uso de las TIC. Revista Universidad y Sociedad del Conocimiento, vol $1 \mathrm{~N}^{\circ} 1$.

Sandín, E (2003) Investigación Cualitativa en Educación. Fundamentos y Tradiciones.

Madrid. Mc Graw and Hill Interamericana.

Santiago, A. (2007). Enseñar y Aprender con nuevos métodos. Revista Universidad Pedagógica Nacional, pg 251.

Sayago, A. (20 de Marzo de 2012). Praxis del Docente Universitario y Trasdisciplinariedad. Barcelona: Universidad Pedagogica Libertador.

Scheaffer, R., \& Mendenhall, W. y. (1986). Elementos de Muestreo. Mexico: Iberoamericana, S.A.

Siegel, Sy 
Castellan, N. (2005). Estadística No Paramétrica. Aplicada a las Ciencias de la Conducta. México: Trillas.Sierra, B. (1984). Epistemología y Sociedad. Madrid España: Editorial Planeta.

Torres, A. (2007). La Autotransformación del Estudiante Universitario, más allá de la formación in tegral. Revis ta Iberoamericana de Educación, 43 (4), 1-9.

Universidad Autónoma de Madrid. (1998). Servicio de Apoyo a la Docencia Universitaria (SADU). Recuperado el 23 de 3 de 2006, de http://www.uam.es/servicios/apoyodocenc ia/ice/sadu/interece.htm\#2

Universidad Bolivariana de Venezuela. (2003) Documento Rector. UBV Caracas

Valderrama Toby y Mena Alejandro. (2006). Rumbo al Socialismo. Barcelona: tercera edición Gobernación del Estado Anzoateguí 